06,05

\title{
Влияние редкоземельных ионов на электрическую поляризацию, индуцированную областями фазового расслоения в $\mathrm{RMn}_{2} \mathrm{O}_{5}$ $(R=\mathrm{Er}, \mathrm{Tb})$
}

\author{
(C) Б.Х. Ханнанов, Е.И. Головенчиц, В.А. Санина \\ Физико-технический институт им. А.Ф. Иофрфе РАН, \\ Санкт-Петербург, Россия \\ "E-mail: sanina@mail.ioffe.ru
}

Поступила в Редакцию 21 октября 2019 г.

В окончательной редакции 21 октября 2019 г.

Принята к публикации 21 октября 2019 г.

\begin{abstract}
Изучено влияние редкоземельных ионов $\left(R=\mathrm{Er}^{3+}, \mathrm{Tb}^{3+}\right)$ с сильной спин-орбитальной связью на диэлектрические свойства и электрическую поляризацию, индуцированную локальными полярными областями фазового расслоения в мультиферроиках $R \mathrm{Mn}_{2} \mathrm{O}_{5}$. Обнаружено качественное отличие этих параметров от изученных ранее в $\mathrm{GdMn}_{2} \mathrm{O}_{5}$, в котором ион $\mathrm{Gd}^{3+}$ в основном состоянии ${ }^{8} S_{7 / 2}$ слабо связан с решеткой. Показано, что свойства полярных областей фазового расслоения, возникающих в подсистеме ионов $\mathrm{Mn}^{3+}$ и $\mathrm{Mn}^{4+}$ благодаря конечной вероятности туннелирования электронов между этими ионами различной валентности, существенным образом зависят от величин кристаллических полей, в которых эти области находятся.
\end{abstract}

Ключевые слова: мультиферроики, зарядовое упорядочение, электрическая поляризация, полярные области фазового расслоения.

DOI: 10.21883/FTT.2020.02.48877.612

\section{1. Введение}

Манганиты $R \mathrm{Mn}_{2} \mathrm{O}_{5}$ ( $R$ - редкоземельный ион, $\left.\mathrm{Bi}\right)$ являются типичными представителями мультиферроиков II-го типа, в которых сегнетоэлектрическое упорядочение индуцируется и управляется магнитным порядком. Характерные значения температур Кюри $T_{C}$ и Нееля $T_{N}$ составляют $30-35$ и $40-45 \mathrm{~K}$, соответственно $[1,2]$. До последнего времени установленным фактом считалось, что $R \mathrm{Mn}_{2} \mathrm{O}_{5}$ характеризуются при комнатной температуре центросимметричной пр. гр. Pbam, которая запрещает существование электрической поляризации. Для объяснения наблюдаемой поляризации в $R \mathrm{Mn}_{2} \mathrm{O}_{5}$ при $T<30-35 \mathrm{~K}$ была предложена модель обменной стрикции. Обменная стрикция возникает при учете зарядового упорядочения пар соседних ионов марганца разной валентности $\left(\mathrm{Mn}^{3+}\right.$ и $\left.\mathrm{Mn}^{4+}\right)$ вдоль оси $b$ с чередующимися ферромагнитным и антиферромагнитным ориентациями спинов этих ионов. Значительное превышение величины ферромагнитного взаимодействия (двойного обмена) над антиферромагнитным (косвенным обменом) приводит к возникновению обменной стрикции, нарушающей центросимметричность решетки и появлению сегнетоэлектрического упорядочения вдоль оси $b$ [3]. Таким образом, считалось, что электрическая поляризация в $R \mathrm{Mn}_{2} \mathrm{O}_{5}$ существует только при температурах $T \leq T_{C}$.

В недавно опубликованной статье [4] сообщалось о структурном исследовании методами резонансной синхротронной рентгеновской дифракции и геометрической оптимизации на основе расчетов из первых принципов для серии кристаллов $R \mathrm{Mn}_{2} \mathrm{O}_{5}$ с различными $R$-ионами при комнатной температуре. Авторы обнаружили наряду с интенсивными рефлексами, соответствующими пр. гр. Pbam, также значительно более слабые рефлексы, которые не могли быть описаны пр.гр. с центральной симметрией. Привлекая физические аргументы, авторы утверждали, что реальная симметрия $R \mathrm{Mn}_{2} \mathrm{O}_{5}$ описывается моноклинной нецентросимметричной группой $P m$, допускающей существование поляризации вдоль оси $b$. Авторы [4] пришли к заключению, что в $R \mathrm{Mn}_{2} \mathrm{O}_{5}$ вплоть до комнатной температуры, должно существовать сегнетоэлектрическое упорядочение иной природы (отличной от обменно-стрикционной). При $T<30-35 \mathrm{~K}$ эти два механизма сосуществуют. Природа высокотемпературного упорядочения в [4] не обсуждалась.

В ряде $R \mathrm{Mn}_{2} \mathrm{O}_{5}(R=\mathrm{Eu}, \mathrm{Gd}, \mathrm{Bi})$ нами изучалась температурная эволюция диэлектрических свойств (диэлектрической проницаемости и проводимости) в широком интервале температур 5-350 K [5-9]. Были установлены два факта. Во-первых, характерные для фазового перехода в упорядоченное сегнетоэлектрическое состояние бездисперсионные максимумы диэлектрической проницаемости и диэлектрических потерь наблюдались вдоль оси $b$ только вблизи $T=T_{C}=30-35 \mathrm{~K}$. В интервале температур 35-350 K таких максимумов не наблюдалось вдоль всех осей. Это свидетельствовало о том, что второго высокотемпературного фазового перехода в упорядоченное сегнетоэлектрическое состояние в этом температурном интервале не возникает. Во-вторых, в параэлектрическом состоянии вплоть до $350 \mathrm{~K}$ наблюда- 
лись зависящие от частоты аномалии диэлектрической проницаемости и проводимости, характерные для локальных полярных областей. Расщепление Брэгговских пиков на два рефлекса [5-9] свидетельствовало о том, что размеры этих областей были достаточны для того, чтобы в них возникало иное, чем в матрице структурное упорядочение.

Характерной особенностью $R \mathrm{Mn}_{2} \mathrm{O}_{5}$ является наличие равного количества ионов марганца $\mathrm{Mn}^{3+}$ (содержащих три $t_{2 g}$ и один $e_{g}$ электрон на $3 d$-оболочке) и $\mathrm{Mn}^{4+}$ (с тремя $t_{2 g}$ электронами на $3 d$-оболочке), что обеспечивает условия для появления диэлектрического зарядового упорядочения. Ионы $\mathrm{Mn}^{4+}$ имеют октаэдрическое кислородное окружение и расположены в слоях с $z=0.25 c$ и $(1-z)=0.75 c$. Ионы $\mathrm{Mn}^{3+}$ имеют нецентральное локальное окружение в виде пятиугольных пирамид и расположены в слоях с $z=0.5 c$. Ионы $R^{3+}$ с окружением подобным $\mathrm{Mn}^{3+}$ расположены в слоях с $z=0$ [10]. Зарядовое упорядочение и конечная вероятность переноса $e_{g}$ электронов между парами ионов $\mathrm{Mn}^{3+}-\mathrm{Mn}^{4+}$ (двойной обмен $[11,12]$ ) являются ключевыми факторами, определяющими электрические полярные состояния $R \mathrm{Mn}_{2} \mathrm{O}_{5}$ при всех температурах. Как отмечалось выше, низкотемпературное сегнетоэлектрическое состояние при $T \leq T_{C}$ обусловлено зарядовым упорядочением вдоль оси $b$ [3]. Перенос же $e_{g}$ электронов между парами ионов $\mathrm{Mn}^{3+}-\mathrm{Mn}^{4+}$, расположенными в соседних слоях, перпендикулярных оси $c$, приводит к образованию локальных областей фазового расслоения с иным распределением ионов $\mathrm{Mn}^{3+}$ и $\mathrm{Mn}^{4+}$ по сравнению с исходной матрицей кристалла. Как показано в работах [5-9] эти локальные области в $\mathrm{RMn}_{2} \mathrm{O}_{5}$ являются полярными и существуют от самых низких температур до температур выше комнатной.

Локальные полярные области формируются в $R \mathrm{Mn}_{2} \mathrm{O}_{5}$, содержащих ионы $\mathrm{Mn}^{3+}$ и $\mathrm{Mn}^{4+}$, за счет процессов фазового расслоения, аналогично манганитам $\mathrm{LaAMnO}_{3} \quad(A=\mathrm{Sr}, \mathrm{Ca}, \mathrm{Ba}) \quad[12,13] . \quad$ Изолированные области фазового расслоения образуются в матрице исходного кристалла за счет процессов самоорганизации, обусловленных конечной вероятностью туннелирования $e_{g}$ электронов между парами ионов $\left(\mathrm{Mn}^{3+}-\mathrm{Mn}^{4+}\right)$ и существуют от самых низких температур до температур выше комнатной. При низких температурах $\left(T<T_{C} \approx 30-40 \mathrm{~K}\right)$ локальные области фазового расслоения в $R \mathrm{Mn}_{2} \mathrm{O}_{5}$ представляют собой изолированные 1D-сверхрешетки ферромагнитных слоев, содержащих ионы $\mathrm{Mn}^{3+}$ и $\mathrm{Mn}^{4+}$ в различных соотношениях. В них наблюдался набор ферромагнитных резонансов [14-16] и электрическая поляризация [7-9] в направлениях магнитного и электрического полей соответственно. При температурах выше $180 \mathrm{~K}$ возникает взаимодействие между ранее изолированными локальными полярными областями, формирующее 2D-сверхструктуры, перпендикулярные оси $c$. В таких сверхструктурах чередовались слои исходной матрицы и областей фазового расслоения. Ширины слоев при комнатной температуре достигали 700-900 $\AA[5,6]$.

При исследовании электрической поляризации, индуцированной локальными полярными областями в $R \mathrm{Mn}_{2} \mathrm{O}_{5} \quad(R=\mathrm{Gd}, \mathrm{Bi}) \quad$ [7-9], было показано, что в этих мультиферроиках области с локальной проводимостью являются областями фазового расслоения и обладают локальным сегнетоэлектрическим упорядочением. Полярность областей фазового расслоения в $R \mathrm{Mn}_{2} \mathrm{O}_{5}$ обусловлена следующими двумя факторами. Внутри областей фазового расслоения двойной обмен, связанный с переносом $e_{g}$-электронов между парами ионов $\mathrm{Mn}^{3+}-\mathrm{Mn}^{4+}$, приводит к тому, что в позициях ионов $\mathrm{Mn}^{4+}$ (кислородных октаэдрах) оказываются янтеллеровские ионы $\mathrm{Mn}^{3+}$, которые деформируют эти октаэдры. В свою очередь, меньшие по размеру ионы $\mathrm{Mn}^{4+}$ (по сравнению с ионами $\mathrm{Mn}^{3+}$ ) оказываются в нецентральных пятиугольных пирамидах и также добавочно их искажают. Оба эти фактора приводят к нецентросимметричности областей фазового расслоения и к их полярности.

Эти области формируют суперпараэлектрическое состояние, которое ниже некоторых температур находится в замороженном суперпараэлектрическом состоянии. Отклик локальных полярных областей в таком состоянии на приложенное электрическое поле имеет вид петель гистерезиса электрической поляризации с остаточной поляризацией. Как показано в работах [7-9] замороженное суперпараэлектрическое состояние существует до температур, при которых кинетическая энергия свободных носителей заряда сравнивается с высотой барьеров на границах областей.

Замороженное суперпараэлектрическое состояние для локальных сегнетоэлектрических областей в диэлектрической центросимметричной матрице было рассмотрено ранее теоретически [17], но экспериментально впервые было обнаружено в $R \mathrm{Mn}_{2} \mathrm{O}_{5}(R=\mathrm{Gd}, \mathrm{Bi})$ [7-9]. При этом было показано, что локальные полярные области фазового расслоения в $R \mathrm{Mn}_{2} \mathrm{O}_{5}$ являются мультиферроидными, так как управляются внешними электрическим и магнитными полями и существуют до температур, значительно превышающих $T_{C}$ низкотемпературного сегнетоэлектрического упорядочения. В этих работах полагалось, что слабо интенсивные нецентральные рефлексы в структурном исследовании при комнатной температуре в [4] относятся к таким областям.

Основной задачей настоящего исследования является экспериментальное обнаружение и изучение свойств электрической поляризации, индуцированной областями фазового расслоения, в $R \mathrm{Mn}_{2} \mathrm{O}_{5}(R=\mathrm{Er}$ и $\mathrm{Tb})$ и сравнение их свойств с ранее изученными $\mathrm{GdMn}_{2} \mathrm{O}_{5}$ [7-9]. Выбранный набор кристаллов обусловлен следующими соображениями. Ион $\mathrm{Gd}^{3+}$ (основное состояние ${ }^{8} S_{7 / 2}$ ) имеет максимальный спин среди $R$-ионов и в основном $S$-состоянии слабо связан с решеткой, так как у него отсутствует спин-орбитальное взаимодействие. Основ- 
ное состояние ионов $\mathrm{Tb}^{3+}\left({ }^{7} F_{6}, S=3, L=3\right)$ характеризуется большим магнитным моментом $\left(J=9.7 \mu_{\mathrm{B}}\right)$, в который вносят вклад как спиновый, так и орбитальный моменты $(S=3, L=3$ соответственно) и имеется сильная спин-орбитальная связь. Ионы $\mathrm{Tb}^{3+}$ описываются в крайне сильном анизотропном изинговском приближении, жестко фиксирующем ориентацию их моментов в плоскости $a b[18]$. Основное состояние иона $\mathrm{Er}^{3+}\left({ }^{4} I_{15 / 2}\right.$, $S=3 / 2, L=6)$ также обладает большим магнитным моментом $\left(J=9.6 \mu_{\mathrm{B}}\right)$, в который основной вклад вносит орбитальный момент и имеется сильное электрическое кристаллическое поле, которое жестко ориентирует моменты ионов $\mathrm{Er}$ вдоль оси $c$ сильной одно-ионной анизотропией [18]. Магнитные моменты $R$-ионов связаны обменным взаимодействием с ионами Мn. Кроме того, $R$-ионы сильно изменяют кристаллическое поле, в котором оказывается подсистема ионов Mn. Представляет интерес сравнить влияния кристаллических полей на свойства Мn-подсистемы при учете спин-орбитального взаимодействия ионов $R^{3+}$.

\section{2. Объекты и методы исследования}

Монокристаллы $R \mathrm{Mn}_{2} \mathrm{O}_{5}(R=\mathrm{Er}, \mathrm{Tb})$ выращивались методом спонтанной кристаллизации из раствора-расплава $[19,20]$. Они имели форму пластинок толщиной $2-3 \mathrm{~mm}$ и площадью $3-5 \mathrm{~mm}^{2}$.

Для измерения диэлектрических свойств и поляризации изготавливались плоские конденсаторы толщиной $0.3-0.6 \mathrm{~mm}$ и площадью $3-4 \mathrm{~mm}^{2}$. Диэлектрическая проницаемость и проводимость определялись с помощью измерителя импеданса Good Will LCR-819 в частотном интервале $0.5-50 \mathrm{kHz}$, в интервале температур $5-330 \mathrm{~K}$.

Электрическая поляризация измерялась $P U N D$ (Positive Up Negative Down) методом [21-23]. Нами использовался адаптированный к измерениям поляризации локальных полярных областей с локальной проводимостью $P U N D$-метод, описанный в [7-9]. PUND-метод позволяет корректно учесть вклад проводимости в измеряемую петлю электрической поляризации. В этом методе отклик внутренней поляризации $P$ может быть выделен при приложении серии положительных $P 1-P 2$ и отрицательных $N 1-N 2$ импульсов напряжения к измеряемому образцу. При этом регистрируются независимые кривые $(P 1-P 2)$ и $(N 1-N 2)$ отклика эффективного изменения поляризации на эту серию импульсов. $P U N D$-метод основан на различии динамики откликов внутренней поляризации и проводимости на импульсы приложенного электрического поля $E$. При выключении импульса поляризация релаксирует значительно медленнее, чем проводимость. Временнб́е интервалы между импульсами $P 1-P 2$ и $N 1-N 2$ должны быть такими, чтобы за это время внутренняя поляризация слабо изменилась, в то время как релаксация проводимости завершилась полностью. В обычных объ- емных сегнетоэлектриках с доменной структурой такие интервалы могут достигать нескольких секунд. В случае отклика от динамических полярных областей фазового расслоения, которые довольно быстро восстанавливают свое равновесное состояние после отключения поля $E$, необходимо сокращать интервалы между импульсами $P 1-P 2$ и $N 1-N 2$. Но при этом по-прежнему необходимо обеспечивать выполнение условия, чтобы за это время внутренняя поляризация слабо изменилась, в то время как релаксация проводимости завершилась полностью. Истинная внутренняя поляризация вычисляется вычитанием парных импульсов $P 1-P 2$ и $N 1-N 2$ друг из друга.

\section{3. Экспериментальные данные и их анализ}

\section{1. Диэлектрические свойства и электрическая поляризация в $\mathrm{ErMn}_{2} \mathrm{O}_{5}$}

На рис. $1, a-f$ приведены температурные зависимости диэлектрической проницаемости $\varepsilon^{\prime}$ для ряда частот вдоль осей $a, b, c$ (рис. $1, a, c, e$ соответственно) и проводимости $\sigma$ вдоль этих же осей (рис. $1, b, d, f$ соответственно). На вставках рисунков для проводимости приведены температурные зависимости относительной локальной проводимости $\sigma_{\mathrm{loc}}=\left(\sigma_{\mathrm{AC}}-\sigma_{\mathrm{DC}}\right) / \sigma_{\mathrm{DC}}$ для соответствующих осей. Согласно принятой картине дисперсии проводимости неоднородных сред, содержащих локальные нанообласти в однородной матрице [24], бездисперсионная низкочастотная проводимость характеризует сквозную проводимость матрицы $\sigma_{\mathrm{DC}}$. Зависящая же от частоты проводимость $\sigma_{\mathrm{AC}}$ (чем выше частота, тем выше проводимость) характеризует локальную проводимость отдельных областей. Соотношение локальной и сквозной проводимостей удобно характеризовать относительной локальной проводимостью $\sigma_{\mathrm{loc}}=\left(\sigma_{\mathrm{AC}}-\sigma_{\mathrm{DC}}\right) / \sigma_{\mathrm{DC}}[24]$.

Мы имеем дело с действительной частью проводимости $\sigma_{1}=\omega \varepsilon^{\prime \prime} \varepsilon_{0}$ [24], которая вычисляется из диэлектрических потерь $\varepsilon^{\prime \prime}$ (измеряется тангенс диэлектрических потерь $\left.\operatorname{tg} \delta=\varepsilon^{\prime \prime} / \varepsilon^{\prime}\right)$. Здесь $\omega-$ угловая частота, а $\varepsilon_{0}$ - диэлектрическая проницаемость вакуума. Проводимость $\sigma_{1}$, обозначаемая в дальнейшем $\sigma$, зависит как от частоты, так и от температуры. Мы полагаем, что в нашем случае локальная проводимость обусловлена областями фазового расслоения, а сквозная проводимость относится к исходной матрице $\mathrm{ErMn}_{2} \mathrm{O}_{5}$.

Только вдоль оси $b$ вблизи $T_{C}=37 \mathrm{~K}$ наблюдается бездисперсионный максимум диэлектрической проницаемости, свидетельствующий о низкотемпературном сегнетоэлектрическом фазовом переходе (вставка к рис. 1,c). Вдоль всех направлений в интервале температур $5-150 \mathrm{~K}$ имеется независящая от температуры диэлектрическая проницаемость $\varepsilon^{\prime}=20$. На этом фоне вдоль оси $a$ начинается рост $\varepsilon^{\prime}$ при температурах $T>150 \mathrm{~K}$ и имеются два скачка такого нарастания при температурах 150 и $250 \mathrm{~K}$ (рис. $1, a$ ). Вдоль осей $b$ и $c$ 

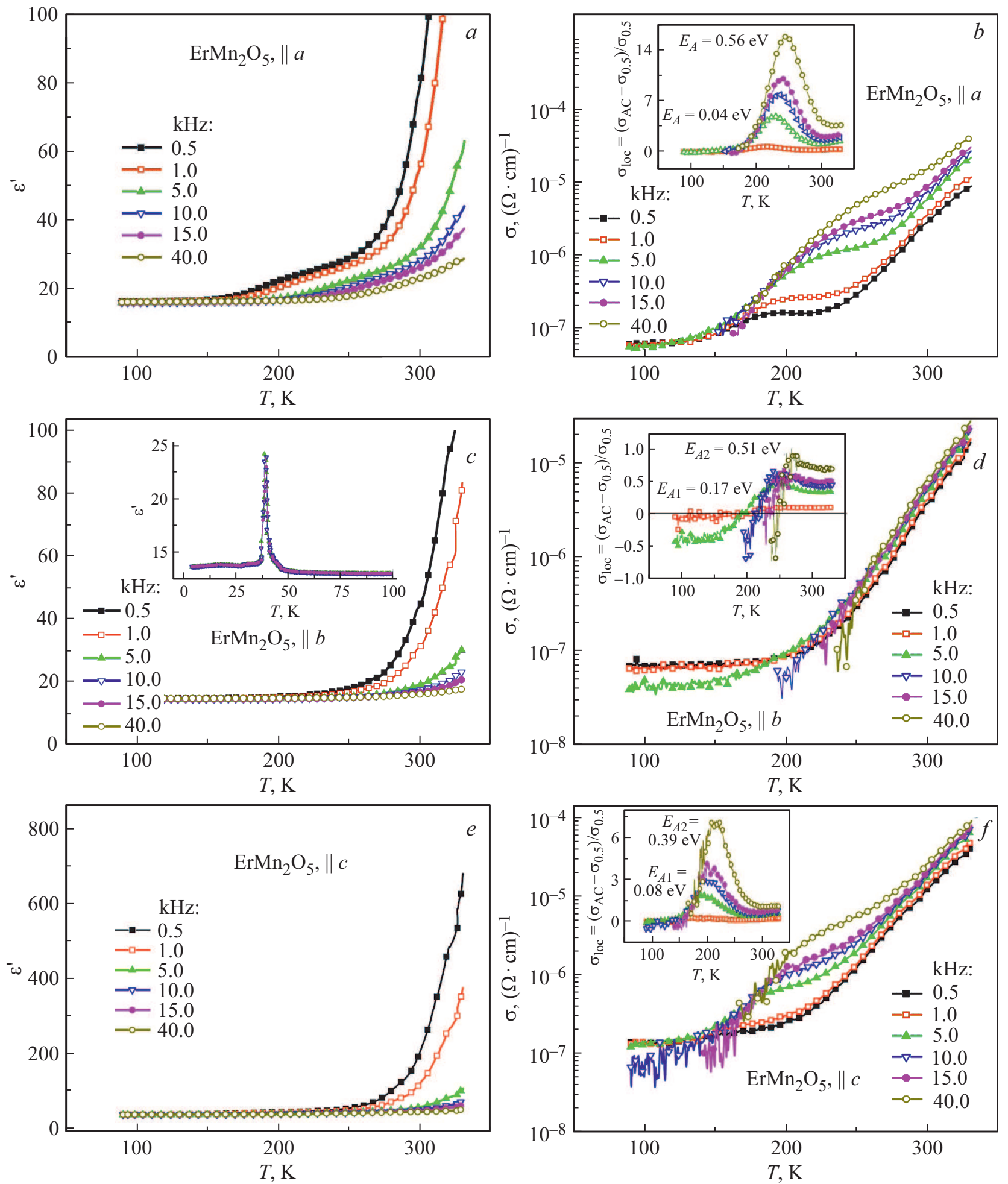

Рис. 1. Температурные зависимости диэлектрической проницаемости $\varepsilon^{\prime}$ для ряда частот вдоль осей $a, b, c$ (рис. $1, a, c, e$ соответственно) и проводимости $\sigma$ вдоль этих же осей (рис. $1, b, d$ и $f$ соответственно) для $\operatorname{ErMn}_{2} \mathrm{O}_{5}$. На вставках к рис. $1, b, d$ и $f$ приведены температурные зависимости локальной проводимости. Частоты указаны на рисунках.

имеется лишь одно нарастание с температурой величины $\varepsilon^{\prime}$ начиная с $T \sim 225 \mathrm{~K}$ (рис. $1, c, e$ соответственно).

Из приведенных на рис. $1, b, d$ и $f$ проводимостей вдоль осей $a, b, c$ в интервале температур 80-330 К видно, что имеются два интервала температур, в которых изменяется характер частотной дисперсии проводимости. При низких температурах вплоть до некоторых температур, зависящих от ориентации осей кристалла, 

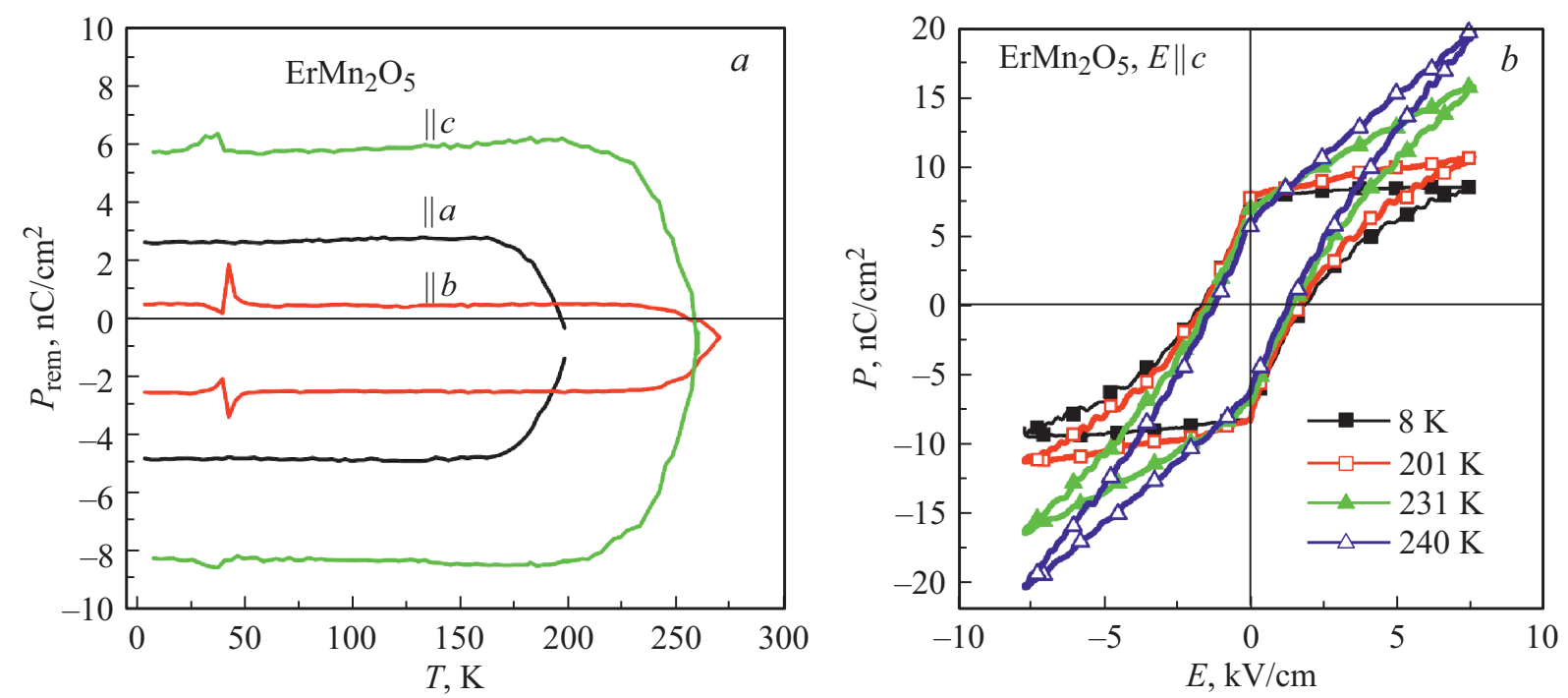

Рис. 2. Температурные зависимости остаточной поляризации $P_{\text {rem }}$ вдоль различных осей $\operatorname{ErMn}_{2} \mathrm{O}_{5}$ (рис. 2,a) и набор петель гистерезиса электрической поляризации при различных температурах вдоль оси с $\mathrm{ErMn}_{2} \mathrm{O}_{5}$ (рис. 2,b).

с ростом частоты проводимость уменьшается, т.е. при этих температурах $\sigma_{\mathrm{DC}}>\sigma_{\mathrm{AC}}$. При нарастании температуры величины $\sigma_{\mathrm{AC}}$ увеличиваются и при неких температурах, на различных частотах, пересекают независящую от температуры и не имеющую частотной дисперсии низкочастотную проводимость. Чем выше частоты, тем при более высоких температурах наблюдаются такие пересечения. Температуры, зависящие от частоты, при которых $\sigma_{\mathrm{AC}}$ сравниваются с $\sigma_{\mathrm{DC}}$, подчиняются закону Аррениуса $\omega=\omega_{0} \exp \left(-E_{A} / k T_{m}\right)$, (где $\omega-$ частота, a $T_{m}-$ температура, при которой $\sigma_{\mathrm{AC}}=\sigma_{\mathrm{DC}}, E_{A}-$ энергия активации на границах локальных областей). Это дает возможность определить активационные барьеры на границах таких низкотемпературных локальных областей для различных осей кристалла (смотри вставки к рис. $1, b, d$ и $f$ ). Как видно из этих же рисунков, вдоль всех осей имеются добавочные высокотемпературные максимумы $\sigma_{\text {loc }}$, смещения температур которых в зависимости от частоты также подчиняются закону Аррениуса, что позволяет определить активационные барьеры на границах этих высокотемпературных локальных областей. Они также указаны на графиках для локальных проводимостей. Таким образом, наблюдение локальной проводимости вдоль всех главных осей $\mathrm{ErMn}_{2} \mathrm{O}_{5}$ с различными активационными барьерами свидетельствует о наличии областей фазового расслоения двух типов.

Мы относим области с отрицательной локальной проводимостью $\sigma_{\mathrm{loc}}$ (т. е. с низкочастотной сквозной бездисперсионной проводимостью $\sigma_{\mathrm{DC}}$, превышающей высокочастотные $\sigma_{\mathrm{AC}}$ проводимости) к локальным областям фазового расслоения, формирующимся внутри марганцевой подсистемы, находящейся при низких температурах под высокими барьерами решетки, обусловленными ионами $\mathrm{Er}^{3+}$ с сильной спин-орбитальной связью. Как было показано в $[5,9,14-16]$ для $R \mathrm{Mn}_{2} \mathrm{O}_{5}$
$(R=\mathrm{Eu}, \mathrm{Gd}, \mathrm{Bi})$ при низких температурах области фазового расслоения занимают малый объем кристалла. С ростом температуры, при увеличивающихся с ростом частоты температурах, увеличивается вероятность для носителей заряда преодолевать высокие барьеры в решетке, обусловленные ионами $\mathrm{Er}^{3+}$. При этом формируются высокотемпературные локальные области фазового расслоения (рис. $1, b, d$ и $f$ ). Отметим, что проводимость в этих областях также обусловлена переносами $e_{g}$ электронов между ионами марганца различной валентности, но уже в областях с более высокими барьерами. Таким образом локальные области фазового расслоения Mn подсистемы обладают различными свойствами при достаточно низких температурах и при более высоких температурах после преодоления решеточного барьера. Основными характеристиками этих областей являются температурные зависимости локальных проводимостей $\sigma_{\mathrm{loc}}$ (вставки на рис. $1, b, d$ и $f$ ) и электрической поляризации (рис. 2, $a$ и $b$ ).

В работах [7-9] для $\mathrm{GdMn}_{2} \mathrm{O}_{5}$ и $\mathrm{BiMn}_{2} \mathrm{O}_{5}$ было показано, что замороженное суперпараэлектрическое состояние, для которого наблюдались петли электрической поляризации, существует до температуры, при которой выполняется условие $\sigma_{\text {loc }} \approx 0$. Условие $\sigma_{\text {loc }} \approx 0$ выполняется при температурах, при которых $k T \approx E_{A}$ активационному барьеру на границах областей. При более высоких температурах области фазового расслоения $\mathrm{Mn-подсистемы} \mathrm{продолжают} \mathrm{существовать,} \mathrm{находясь} \mathrm{в}$ обычном суперпараэлектрическом состоянии, для которого электрическая поляризация исчезает.

В ErMn $\mathrm{O}_{5}$ вдоль оси $a$ вблизи $190-200 \mathrm{~K}$ (рис. $\left.1, b\right)$ происходит скачкообразное увеличение сквозной проводимости матрицы (проводимости на самой низкой частоте). Естественно принять, что оно обусловлено термоактивацией носителей заряда из низкотемпературных 
областей фазового расслоения при выполнении условия $k T \approx E_{A} \approx 0.04 \mathrm{eV}$ активационных барьеров этих областей. Из рис. $1, b$ видно, что при этих же температурах усиленная сквозная проводимость матрицы приближается по величине к значениям совпадающих между собой проводимостей $\sigma_{\mathrm{AC}}$. При этом выполняется условие $\sigma_{\text {loc }} \sim 0$ на всех частотах, при котором локальные полярные области изменяют состояние от замороженного суперпараэлектрического к обычному суперпараэлектрическому и электрическая поляризация областей фазового расслоения резко уменьшается до нуля (рис. 2,a). Вдоль оси $b$ активационный барьер на границах низкотемпературных областей фазового расслоения в $\mathrm{ErMn}_{2} \mathrm{O}_{5}$ значительно выше $(0.18 \mathrm{eV})$ и сближение величин нарастающей сквозной проводимости матрицы и совпадающих $\sigma_{\mathrm{AC}}$ высокотемпературных областей фазового расслоения происходит вблизи $270 \mathrm{~K}$ (рис. $1, d$ ). При этом во всем диапазоне температур до $270 \mathrm{~K}$ величины $\sigma_{\text {loc }}$ малы (вставка к рис. $1, d$ ), что уменьшает величину наблюдаемой поляризации вдоль оси $b$ (рис. 2,a). Вдоль оси с при температуре $175 \mathrm{~K}$ совпадающие по величине $\sigma_{\mathrm{AC}}$ проводимости высокотемпературных областей фазового расслоения сильно отличаются от сквозной проводимости матрицы (рис. $1, f)$. Сближение этих величин начинается с $200 \mathrm{~K}$ и при температуре $260 \mathrm{~K}$ величины $\sigma_{\mathrm{loc}}$ и поляризация приближаются к нулю (вставка к рис. $1, f$ и $2, a$ ).

Обратимся теперь к рассмотрению петель гистерезиса электрической поляризации в $\mathrm{ErMn}_{2} \mathrm{O}_{5}$, индуцированной локальными полярными областями фазового расслоения. На рис. 2, а представлены температурные зависимости остаточной поляризации вдоль различных осей $\mathrm{ErMn}_{2} \mathrm{O}_{5}$. Видно, что максимальная остаточная поляризация наблюдается вдоль оси $c$ до температуры $260 \mathrm{~K}$. Минимальная поляризация возникает вдоль оси $b$ и существует до температуры $270 \mathrm{~K}$. Вдоль оси $a$ имеется промежуточная по величине поляризация, наблюдающаяся до $T<190 \mathrm{~K}$. На рис. $2, b$ для примера показан набор петель гистерезиса для ряда температур вдоль оси $c$. Видно, что вид и величина $P_{\text {rem }}^{c}$ в интервале температур 8-200 K изменяются мало, а в интервале температур 200-260K довольно резко уменьшаются. Отметим, что вид петель гистерезиса вдоль всех осей подобен, а о величинах поляризации и температурах их существования можно судить по рис. $2, a$.

Сопоставление данных рис. 2, $a$ и 1 для диэлектрической проницаемости и проводимостей позволяет сделать заключения о природе наблюдаемых поляризаций вдоль различных осей $\mathrm{ErMn}_{2} \mathrm{O}_{5}$. Действительно, наблюдаемую нами электрическую поляризацию формируют именно локальные полярные области фазового расслоения. Выбор высокочастотного режима $P U N D$-метода измерения петель гистерезиса, а также температурный интервал их существования предполагают, что отклик на приложенные импульсы электрического поля дают области с высокой динамикой. Это локальные полярные области фазового расслоения и флуктуационная область вблизи фазового перехода в сегнетоэлектрическое упорядочение [7-9].

Наблюдается четкая корреляция между диэлектрическими свойствами, обусловленными областями фазового расслоения, и параметрами наблюдаемой электрической поляризации. Сопоставление рис. 1 и 2 для соответствующих направлений кристалла показывает, что имеется оптимальное соотношение активационных барьеров областей фазового расслоения $\mathrm{Mn}$-подсистемы под барьерами, обусловленными ионами $\mathrm{Er}^{3+}$, и после преодоления этих барьеров. Имеется также и оптимальная величина локальной проводимости для максимальной поляризации, существующей до высокой температуры. Как отмечено выше, максимальная поляризация $\left(\approx 7.5 \mathrm{nC} / \mathrm{cm}^{2}\right)$ наблюдается до температуры $260 \mathrm{~K}$ вдоль оси $c$. При этом отношение активационных барьеров двух типов областей $(0.39 / 0.08 \approx 5)$, величина $\sigma_{\text {loc }} \approx 7$ для высокотемпературного максимума. Эти параметры можно считать оптимальными для $\mathrm{ErMn}_{2} \mathrm{O}_{5}$. Минимальная поляризация $\left(\approx 2 \mathrm{nC} / \mathrm{cm}^{2}\right)$ наблюдается до температуры $270 \mathrm{~K}$ вдоль оси $b$ (рис. 2, $a$ ). При этом отношение активационных барьеров двух типов областей $(0.51 / 0.18 \approx 2.8)$, величина $\sigma_{\text {lос }}$ при этом $<1$. Вдоль оси а отношение активационных барьеров двух типов областей $(0.56 / 0.04 \approx 14)$, величина $\sigma_{\text {loc }} \approx 17$ для высокотемпературного максимума. При этом поляризация $\left(\approx 4 \mathrm{nC} / \mathrm{cm}^{2}\right)$ наблюдается до сравнительно низкой температуры $190 \mathrm{~K}$. В результате, мы приходим к заключению, что близость активационных барьеров двух типов локальных областей существенно уменьшает локальную проводимость и поляризацию, но обеспечивает существование поляризации до более высокой температуры (вдоль оси $b$ ). Слишком большая разница активационных барьеров вдоль оси $a$ приводит к резкому росту $\sigma_{\text {loc }}$ для высокотемпературного максимума. В результате внутри таких локальных областей появляются избыточные (не связанные перезарядкой пар ионов $\mathrm{Mn}^{3+}-\mathrm{Mn}^{4+}$ ) носители под барьером, экранирующие барьер, уменьшающие поляризацию, что понижает и температуру существования поляризации (ось $a$ ). Отметим, что вдоль оси $a$ на температурной зависимости диэлектрической проницаемости $\varepsilon^{\prime}$ возникают добавочные более низкотемпературные скачки, зависящие от частоты, которые мы относим к появлению сравнительно низкотемпературного замороженного суперпараэлектрического состояния, дающего вклад в поляризацию областей фазового расслоения. Более высокотемпературные области локальной проводимости относятся к обычному суперпараэлектрическому состоянию, не дающему вклад в петли электрической поляризации. В результате, температуры исчезновения электрической поляризации на температурных зависимостях диэлектрической проницаемости (рис. 1, $a, c, e$ ) совпадают с самыми низкотемпературными нарастаниями величин $\varepsilon^{\prime}$, зависящими от частоты. 

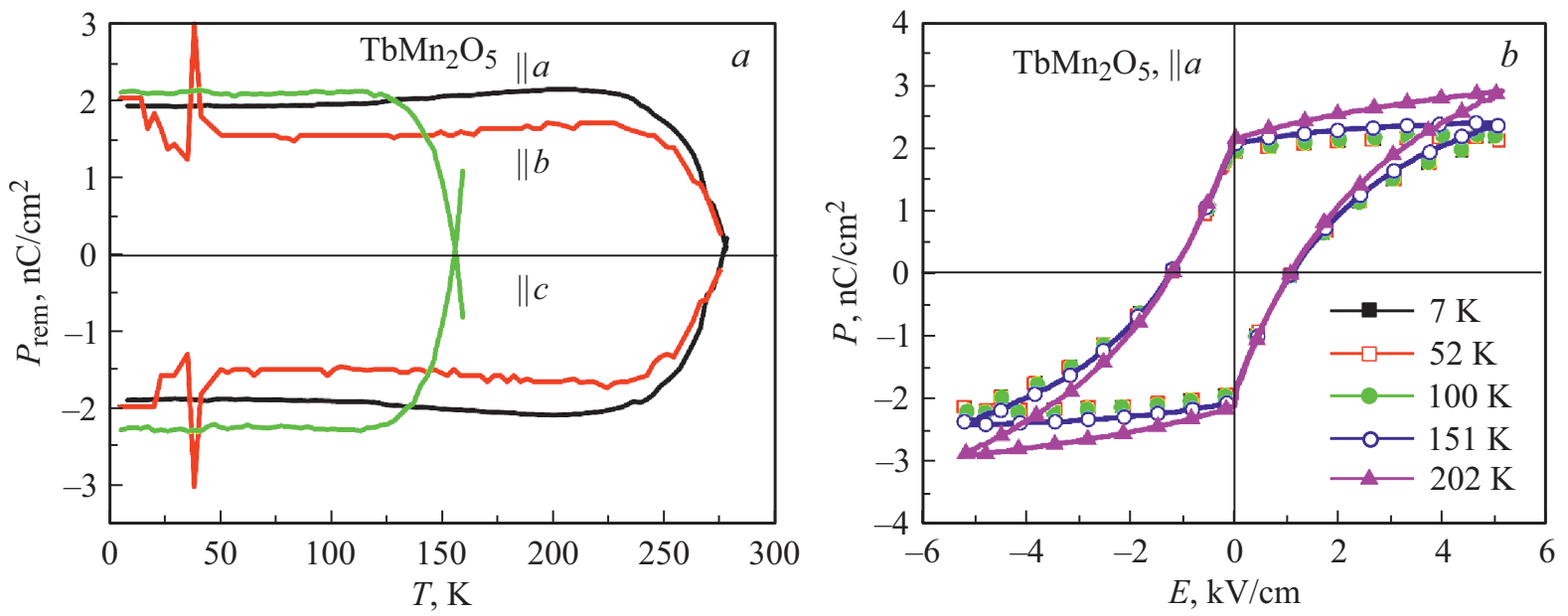

Рис. 3. Температурные зависимости остаточной поляризации $P_{\text {rem }}$ вдоль различных осей $\mathrm{TbMn}_{2} \mathrm{O}_{5}$ (рис. $\left.3, a\right)$ и набор петель гистерезиса электрической поляризации при различных температурах вдоль оси $a \mathrm{TbMn}_{2} \mathrm{O}_{5}$ (рис. $3, b$ ).

\section{2. Диэлектрические свойства и электрическая поляризация в $\mathrm{TbMn}_{2} \mathrm{O}_{5}$}

Учитывая приведенный выше анализ корреляции данных диэлектрических свойств и поляризации для $\mathrm{ErMn}_{2} \mathrm{O}_{5}$, мы прежде всего приведем для $\mathrm{TbMn}_{2} \mathrm{O}_{5}$ данные для электрической поляризации, а затем проанализируем их диэлектрические свойства для каждой из осей кристалла. При этом мы будем сравнивать ситуации в $\mathrm{TbMn}_{2} \mathrm{O}_{5}$ и $\mathrm{ErMn}_{2} \mathrm{O}_{5}$ вдоль соответствующих осей.

На рис. 3, $a$ и $b$ приведены температурные зависимости остаточной поляризации вдоль различных осей $\mathrm{TbMn}_{2} \mathrm{O}_{5}$ и петли гистерезиса электрической поляризации вдоль оси $a$. Видно, что в отличие от $\mathrm{ErMn}_{2} \mathrm{O}_{5}$ (рис. 2,a), величины остаточной поляризации вдоль всех осей в $\mathrm{TbMn}_{2} \mathrm{O}_{5}$ близки по величине, и вдоль осей $a$, и особенно оси $c$, значительно меньше чем в $\operatorname{ErMn}_{2} \mathrm{O}_{5}$. Вдоль оси $b$ величины остаточной поляризации в этих двух кристаллах близки. Мы относим это за счет значительно большей величины сильно связанного с решеткой орбитального момента ионов $\mathrm{Er}^{3+}$ (по сравнению с ионами $\mathrm{Tb}^{3+}$ ). При этом, как отмечалось выше, момент в $\mathrm{ErMn}_{2} \mathrm{O}_{5}$ направлен преимущественно вдоль оси $c . \mathrm{B} \mathrm{TbMn}_{2} \mathrm{O}_{5}$ орбитальный момент ориентирован в плоскости $a b$.

На рис. 4, a, c, $e$ приведены температурные зависимости диэлектрической проницаемости $\varepsilon^{\prime}$ для ряда частот в $\mathrm{TbMn}_{2} \mathrm{O}_{5}$ вдоль осей $a, b$, и $c$ соответственно. На рис. $4, b, d$ и $f$ представлены температурные зависимости проводимости для тех частот, вдоль тех же осей. На вставках к этим рисункам приведены соответствующие температурные зависимости $\sigma_{\mathrm{loc}}=\left(\sigma_{\mathrm{AC}}-\sigma_{\mathrm{DC}}\right) / \sigma_{\mathrm{DC}}$. $\mathrm{B} \mathrm{TbMn}_{2} \mathrm{O}_{5}$, как и в $\mathrm{ErMn}_{2} \mathrm{O}_{5}$, вдоль всех осей существуют как низкотемпературные, так и высокотемпературные локальные области фазового расслоения.

Вдоль оси $a$ в $\mathrm{TbMn}_{2} \mathrm{O}_{5}$ проводимость в диапазоне температур 5-275K (рис. 4,b) на порядок меньше, чем в $\mathrm{ErMn}_{2} \mathrm{O}_{5}$ (рис. 1, b). Этот факт определяет качественное изменение всех параметров в $\mathrm{TbMn}_{2} \mathrm{O}_{5}$ по сравнению с $\mathrm{ErMn}_{2} \mathrm{O}_{5}$. Действительно, величина $\varepsilon^{\prime}$ в $\mathrm{TbMn}_{2} \mathrm{O}_{5}$ вдоль оси $a$ (рис. 4,a) значительно меньше, чем в $\mathrm{ErMn}_{2} \mathrm{O}_{5}$ (рис. $\left.1, a\right)$. В результате, первый скачок $\varepsilon^{\prime}$ в $\mathrm{TbMn}_{2} \mathrm{O}_{5}$ вблизи $120 \mathrm{~K}$ лишь обозначает область незначительного роста $P_{\text {rem }}^{a}$ продолжающей существовать до более высокой температуры $275 \mathrm{~K}$ поляризации (рис. 3,a). В $\mathrm{TbMn}_{2} \mathrm{O}_{5}$, как и в $\mathrm{ErMn}_{2} \mathrm{O}_{5}$, вдоль оси $a$ барьеры на границах низкотемпературных областей соизмеримы (0.06 и $0.04 \mathrm{eV}$ соответственно в то время как барьеры на границах высокотемпературных областей в $\mathrm{ErMn}_{2} \mathrm{O}_{5}(0.56 \mathrm{eV})$ (рис. $\left.1, b\right)$ практически в три раза больше, чем в $\mathrm{TbMn}_{2} \mathrm{O}_{5}(0.19 \mathrm{eV})$ (рис. $\left.4, b\right)$. Высокотемпературные локальные области в $\mathrm{TbMn}_{2} \mathrm{O}_{5}$ (как и в $\mathrm{ErMn}_{2} \mathrm{O}_{5}$ ) обладают близкими высокими относительными локальными проводимостями $\left(\sigma_{\mathrm{loc}} \approx 18\right)$, но на фоне отличающихся на порядок величин $\sigma_{\mathrm{AC}}$ и $\sigma_{\text {DC }}$ (меньших в $\mathrm{TbMn}_{2} \mathrm{O}_{5}$ ). В результате в $\mathrm{TbMn}_{2} \mathrm{O}_{5}$ не происходит полной экранировки внутреннего поля высокотемпературных областей вплоть до $275 \mathrm{~K}$, но при значительно ослабленной величине поляризации (рис. 3,a). Напомним, что в $\mathrm{ErMn}_{2} \mathrm{O}_{5}$ экранировка внутреннего поля высокотемпературных областей наблюдалась вблизи $200 \mathrm{~K}$, но при в два раза большей $P_{\mathrm{rem}}^{a}$ (рис. 2,a).

Вдоль оси $b$ у обоих кристаллов проводимости слабо отличаются, и это является определяющим фактором подобия свойств электрической поляризации в $\mathrm{ErMn}_{2} \mathrm{O}_{5}$ и $\mathrm{TbMn}_{2} \mathrm{O}_{5}$ (сравни рис. 2, $a$ и 3,a) вдоль этой оси. Мы связываем это с тем, что во всех $R \mathrm{Mn}_{2} \mathrm{O}_{5}$, независимо от типа $R$-ионов, вдоль оси $b$ чередуются пары ионов $\mathrm{Mn}^{3+}-\mathrm{Mn}^{4+}$, и повышенная проводимость вдоль этой оси обусловлена переносом $e_{g}$ электронов между этими парами ионов. Наблюдаются близкие величины как $P_{\mathrm{rem}}^{b}$, так и температур существования электрической поляризации в $\mathrm{ErMn}_{2} \mathrm{O}_{5}$ и $\mathrm{TbMn}_{2} \mathrm{O}_{5}$. 

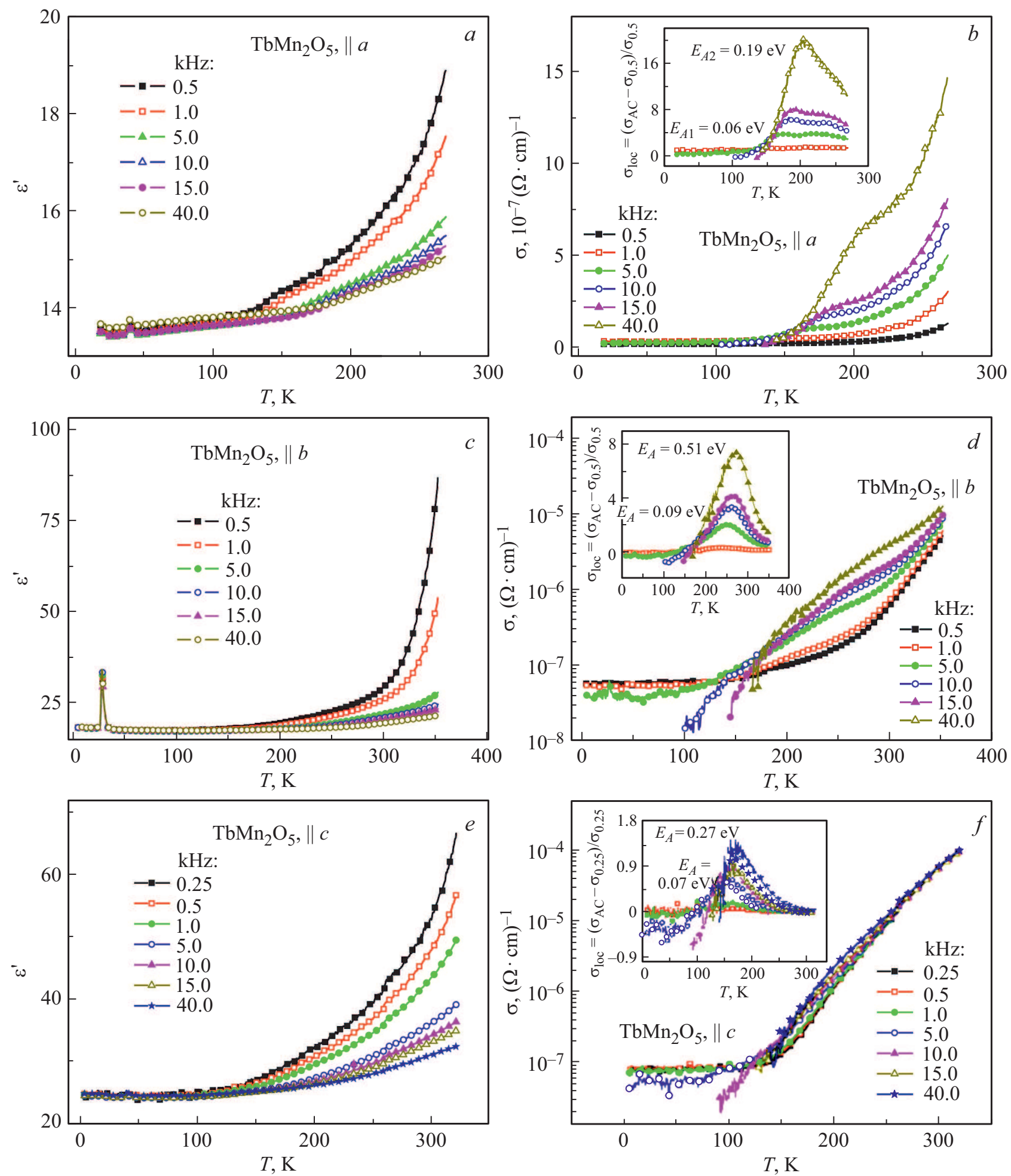

Рис. 4. Температурные зависимости диэлектрической проницаемости $\varepsilon^{\prime}$ для ряда частот вдоль осей $a, b, c$ (рис. 4,a,c,e соответственно) и проводимости $\sigma$ вдоль этих же осей (рис. $4, b, d$ и $f$ соответственно) для $\mathrm{TbMn}_{2} \mathrm{O}_{5}$. На вставках к рис. $4, b, d$ и $f$ приведены температурные зависимости локальной проводимости. Частоты указаны на рисунках.

Проводимости вдоль оси $c$ в $\mathrm{TbMn}_{2} \mathrm{O}_{5}$ и $\mathrm{ErMn}_{2} \mathrm{O}_{5}$ (рис. $4, f$ и $2, f$ соответственно) также близки, что также связано с тем, что во всех $R \mathrm{Mn}_{2} \mathrm{O}_{5}$ при всех температурах образование областей фазового расслоения обусловлено переносом $e_{g}$ электронов между соседними плоскостями, перпендикулярными оси $c$, содержащими только ионы $\mathrm{Mn}^{3+}$ или $\mathrm{Mn}^{4+}$. Однако, диэлектрические проницаемости и локальные проводимости в $\mathrm{TbMn}_{2} \mathrm{O}_{5}$ 
существенно меньше, чем в $\mathrm{ErMn}_{2} \mathrm{O}_{5}$ (сравни рис. 4, $e$ и $1, e$, а также рис. $4, f$ и $1, f$ соответственно), что приводит к качественному отличию величин и температур существования электрических поляризаций. Вдоль оси $c$ в $\mathrm{ErMn}_{2} \mathrm{O}_{5}$ поляризация величиной $\approx 7.5 \mathrm{nC} / \mathrm{cm}^{2}$ существует до температуры $260 \mathrm{~K}$, в то время как в $\mathrm{TbMn}_{2} \mathrm{O}_{5}$ поляризация величиной $\approx 2 \mathrm{nC} / \mathrm{cm}^{2}$ существует до температуры $154 \mathrm{~K}$ (рис. 2, $a$ и $3, a$ соответственно). Это объясняется тем, что ионы $\mathrm{Er}^{3+}$ сильно искажают решетку именно вдоль оси $c$, в то время как ионы $\mathrm{Tb}^{3+}$ оказывают влияние в плоскости $a b$. Как это имело место и в $\mathrm{ErMn}_{2} \mathrm{O}_{5}$, в $\mathrm{TbMn}_{2} \mathrm{O}_{5}$ температурные нарастания диэлектрической проницаемости согласуются с температурами исчезновения электрической поляризации (рис. $3, a$ ).

Обращает на себя внимание тот факт, что самые низкие и довольно близкие температуры существования электрической поляризации наблюдаются для $\mathrm{ErMn}_{2} \mathrm{O}_{5}$ вдоль оси $a$ (рис. $2, b$ ) и $\mathrm{TbMn}_{2} \mathrm{O}_{5}$ вдоль оси $c$ (рис. $4, f)$. В обоих случаях они определяются температурами фазовых превращений при температурах, при которых все высокочастотные проводимости низкотемпературных областей сравниваются между собой на фоне слабых локальных проводимостей высокотемпературных областей (рис. $1, b$ и $4, f$ соответственно). Высокотемпературные области формируют при этом обычное суперпараэлектрическое состояние, для которого нет электрической поляризации. Этот факт также обусловлен тем обстоятельством, что ионы $\mathrm{Er}^{3+}$ и $\mathrm{Tb}^{3+}$ именно вдоль осей $a$ и $c$ соответственно, не искажают решетку. По этой причине в $\mathrm{TbMn}_{2} \mathrm{O}_{5}$ вдоль оси $c$ при $T>150 \mathrm{~K}$ резко нарастает слабо дисперсионная проводимость с малым вкладом локальной проводимости (рис. 4,f). Как указано выше, в $\mathrm{ErMn}_{2} \mathrm{O}_{5}$ вдоль оси $a$ (рис. $\left.1, b\right)$ при $T>190 \mathrm{~K}$, напротив, возникают высотемпературные локальные области с аномально высокой локальной проводимостью внутри этих областей, что приводит к экранировке внутренних электрических полей и электрической поляризации.

Отметим, что существенная разница в поведении локальных проводимостей и электрической поляризации, индуцируемых локальными полярными областями фазового расслоения, в $\mathrm{GdMn}_{2} \mathrm{O}_{5}$ [9-11] и $R \mathrm{Mn}_{2} \mathrm{O}_{5}$ $(R=\mathrm{Er}, \mathrm{Tb})$ обусловлена отсутствием в $\mathrm{GdMn}_{2} \mathrm{O}_{5}$ добавочных барьеров в решетке, формируемых ионами $\mathrm{Er}^{3+}$ и $\mathrm{Tb}^{3+}$ с сильными спин-орбитальными взаимодействиями. В основном состоянии ионов $\mathrm{Gd}^{3+}$ спин-орбитальное взаимодействие отсутствует. В результате в $\mathrm{GdMn}_{2} \mathrm{O}_{5}$ вдоль одних и тех же осей не сосуществуют локальные области двух типов с различными барьерами на их границах. Как показано выше, именно взаимодействие этих двух типов областей в различающихся по величинам кристаллических полях, определяют температурную эволюцию диэлектрических свойств и поляризации в $R \mathrm{Mn}_{2} \mathrm{O}_{5}(R=\mathrm{Er}, \mathrm{Tb})$.

\section{4. Заключение}

Показано, что различные $R$-ионы в $R \mathrm{Mn}_{2} \mathrm{O}_{5}$ качественно изменяют свойства локальных областей фазового расслоения и индуцируемой ими электрической поляризации. Появление областей фазового расслоение обусловлено состояниями Мn-подсистемы (зарядовым упорядочением ионов различной валентности $\left(\mathrm{Mn}^{3+}\right.$ и $\left.\mathrm{Mn}^{4+}\right)$ и их перезарядкой $e_{g}$ электронами). Но эти области находятся в различных кристаллических полях решетки в зависимости от типа $R$-ионов. Ионы $\mathrm{Gd}^{3+}$ в $\mathrm{GdMn}_{2} \mathrm{O}_{5}$ не искажают решетку и наблюдаемые в них локальные полярные области и электрическая поляризация отражают свойства самой Мn-подсистемы. Ионы $\mathrm{Er}^{3+}$ и $\mathrm{Tb}^{3+}$ в $R \mathrm{Mn}_{2} \mathrm{O}_{5}$, напротив сильно и поразному искажают кристаллические поля, в которых находятся ионы $\mathrm{Mn}^{3+}$ и $\mathrm{Mn}^{4+}$ и перезаряжающие их электроны. В результате, в Мn-подсистеме возникают локальные области двух типов. Ниже определенных температур локальные области фазового расслоения $\mathrm{Mn}$ подсистемы находятся под барьером в кристаллическом поле, формируемом $R$-ионами. С ростом температуры, носители заряда начинают преодолевать эти барьеры в решетке, и области фазового расслоения и индуцируемая ими электрическая поляризация изменяют свои свойства. Так как спин-орбитальные взаимодействия для ионов $\mathrm{Er}^{3+}$ и $\mathrm{Tb}^{3+}$ различаются по величине и анизотропии, то диэлектрические свойства и поляризация в $\mathrm{ErMn}_{2} \mathrm{O}_{5}$ и $\mathrm{TbMn}_{2} \mathrm{O}_{5}$ сильно различаются между собой, и оба эти кристалла имеют отличные свойства по сравнению с $\mathrm{GdMn}_{2} \mathrm{O}_{5}[7-9]$.

\section{Финансирование работы}

Авторы благодарны за финансовую поддержку работы Российскому Фонду Фундаментальных Исследований (гранты 18-32-00241, 20-02-0667) и Программе 1.4 Президиума РАН „Актуальные проблемы физики низких температур“.

\section{Конфликт интересов}

У авторов отсутствует конфликт интересов.

\section{Список литературы}

[1] N. Hur, S. Park, P.A. Sharma, J.S. Ahn, S. Guba, S-W. Cheong. Nature (London) 429, 392 (2004).

[2] Y. Noda, H. Kimura, M. Fukunaga, S. Kobayashi, I. Kagomiya, K. Kohn. J. Phys.: Condens. Matter 20, 434206 (2008).

[3] J. van den Brink, D.I. Khomskii. J. Phys.: Condens. Matter 20, 434217 (2008).

[4] V. Baledent, S. Chattopadhyay, P. Fertey, M.B. Lepetit, M. Greenblatt, B. Wanklyn, F.O. Saouma, J.I. Jang, P. FouryLeylekian. Phys. Rev. Lett. 114, 117601 (2015).

[5] V.A. Sanina, E.I. Golovenchits, V.G. Zalesskii, S.G. Lushnikov, M.P. Scheglov, S.N. Gvasaliya, A. Savvinov, R.S. Katiyar, H. Kawaji, T. Atake. Phys. Rev. B 80, 224401 (2009). 
[6] V.A. Sanina, E.I. Golovenchits, B.Kh. Khannanov, M.P. Scheglov, V.G. Zalesskii. Письма в ЖЭТФ 100, 451 (2014).

[7] B.Kh. Khannanov, V.A. Sanina, E.I. Golovenchits, M.P. Scheglov. Письма в ЖЭТФ 103, 274 (2016).

[8] B.Kh. Khannanov, V.A. Sanina, E.I. Golovenchits, M.P. Scheglov. JMMM 421, 326 (2017).

[9] В.А. Санина, Б.Х. Ханнанов, Е.И. Головенчиц, М.П. Щеглов. ФТТ 60, 531 (2018).

[10] P.G. Radaelli, L.C. Chapon. J. Phys.: Condens. Matter 20, 434213 (2008).

[11] P.G. de Gennes. Phys. Rev. 118, 141 (1960).

[12] Л.П. Горьков. УФН 168, 655 (1998).

[13] М.Ю. Каган, К.И. Кугель. УФН 171, 533 (2001).

[14] В.А. Санина, Е.И. Головенчиц, В.Г. Залесский. Письма в ЖЭТФ 95, 429 (2012).

[15] V.A. Sanina, E.I. Golovenchits, V.G. Zalesskii. J. Phys.: Condens. Matter 24, 346002 (2012).

[16] В.А. Санина, Б.Х. Ханнанов, Е.И. Головенчиц. ФТТ 59, 1932 (2017).

[17] M.D. Glinchuk, E.A. Eliseev, A.N. Morozovska. Phys. Rev. B 78, 134107 (2008).

[18] К.П. Белов, А.К. Звездин, А.М. Кадомцева, Р.З. Левитин. „Ориентационные переходы в редкоземельных магнетиках“, Наука, М. (1979).

[19] В.А. Санина, Л.М. Сапожникова, Е.И. Головенчиц, Н.В. Морозов. ФТТ 30, 3015 (1988).

[20] А.В. Бабинский, Е.И. Головенчиц, Н.В. Морозов, Л.М. Сапожникова. ФТТ 34, 108 (1992).

[21] J.F. Scott, L. Kammerdiner, L.M. Parris, S. Traynor, V. Ottenbacher, A. Shawabkeh, W.F. Oliver. J. Appl. Phys. 64, 787 (1988).

[22] M. Fukunaga, Y. Noda. J. Phys. Soc. J. 77, 0647068 (2008).

[23] S.M. Feng, Y.S. Chai, J.L. Zhu, N. Manivannan, Y.S. Oh, L.J. Wang, Y.S. Yang, C.Q. Jin, Kee Hoon Kim. New J. Phys. 12, 073006 (2010).

[24] A.R. Long. Adv. Phys. 31, 587 (1982).

Редактор Т.Н. Василевская 